\title{
A Fast and Economical Photoelastic Model Making of the Teeth and Surrounding Structure
}

\author{
Shetty Prajna $\mathrm{P}^{1^{*}}$, Patil N Konark ${ }^{1}$, Meshramkar Roseline ${ }^{1}$, Nadiger Ramesh $\mathrm{K}^{1}$. \\ ${ }^{I}$ Department of Prosthodontics, SDM college of dental sciences and hospital, Dharwad, Karnataka, India.
}

\begin{abstract}
Photoelastic stress analysis is an experimental technique for stress and strain analysis. It is based on the property of birefringence. Simulation of the accurate geometrical shape and mechanical property of the material to be studied is a mandatory requirement. It has always been a donting task to make an accurate photoelastic model of the dental structure. Innumerable materials and indefinite methods are explained in the literature. This article provides with a simple, cost effective, easily available material and a simple technique for making a photoelastic model.
\end{abstract}

Keywords:-photoelastic model, photoelasticity, stress analysis, epoxy resin teeth.

\section{Introduction}

The photoelastic method was established by Sir David Brewster, a British physicist, and B Zak applied this in the dental research for the first time in 1935 to investigate stress distribution in teeth and alveolar bone. The word photo implies use of light rays and optical techniques while elasticity depicts the study of stresses and deformations in elastic bodies. It is based on the property of some transparent materials to exhibit colourful patterns when viewed with polarised light. The patterns that develop are consequently related to the distribution of internal stresses. Photelasticity is an experimental technique for stress and strain analysis that is particularly useful for members having complicated geometry, complicated loading conditions or both. Photo elastic analysis supplies visual information on the stress distribution. It is limited by the fact that it does not supply information on the state of a single component of sample.

The photoelastic method is based upon a unique property of some transparent materials, in particular, certain plastics. When the model is stressed and a ray of light enters along one of the directions of principal stress, the light is divided into two component waves, each with its plane of vibration (plane of polarization) parallel to one of the remaining two Principal planes (planes on which shear stress is zero). Furthermore, the light travels along these two paths with different velocities, which depend upon the magnitudes of the remaining two principal stresses in the material. The incident light is resolved into components having planes of vibration parallel to the directions of the principal stresses. Since these waves traverse the body with different velocities, the waves emerge with a new phase relationship, or relative retardation. Specifically, the relative retardation is the difference between the numbers of wave cycles experienced by the two rays travelling inside the body. This phenomenon is called double refraction or birefringence, and is the same as exhibited by certain optical crystals - but in photoelasticity the double refraction is artificial, being controlled by the state of stress or strain at each point in the body. The two waves are brought together in the photoelastic polariscope, and permitted to come into optical interference. In the nomenclature of optical interference, the dark and light bands obtained are called fringes, and the fringe order is defined as the value of $\mathrm{N}$ along the band under consideration.

Clinical relevance of the technique: - This article provides an easy and economical technique for the fabrication of a photoelastic model. The material used is easily available. The procedure of manipulation of the material is not very technique sensitive and the result obtained is appreciable.

\section{TECHNIQUE TO MAKE THE PHOTOELASTIC MODEL OF THE TEETH AND DENTAL STRUCTURE}

* A maxillary central incisor is carved according to the dimensions given in wheeler.(FIG 2)

- A sprue is attached to lingual surface of the central incisor at an angle so that the material can be poured easily.

* The tooth is embedded one half into putty. The other is embedded into the other putty. (FIG 3)

* Once set the tooth is removed from the mould and the mould is stabilised.

- An epoxy resin c-51(3222) and k6 hardener is selected to simulate the tooth.

* The resin is mixed in the ratio of 1:10 (1 part of hardener and 10 part of the resin).(FIG 4)

- The resin hardener mixture is slowly dispensed accurately into a broad bowl to prevent the incorporation of air bubbles and mixed with a glass rod. (FIG 5)

* The mixture is mixed slowly along the edges for thirty minutes to get a clear mix

* The photoelastic material is then slowly poured into the mould through the passage obtained due the sprue.

* Once poured the material is allowed to set. 
* In room temperature it is completely cured in three days. It can also be cured at $100^{\circ} \mathrm{c}$ for 24 hours in dry heat in a furnace.(FIG 6)

* Once set, it is removed and the sprue cut and polished.(FIG 7)

* Tin foil is placed on the root surface to standardise the thickness of the periodontal ligament.

* The resin is mixed in the similar way for the simulation of alveolar bone and the simulated tooth is placed into the alveolar bone mixture and stabilised.(FIG 8)

* Once set, the foil is removed from the root and a silicone material is applied in that area to simulate periodontal ligament.

* The tooth model is ready for photoelastic test.

\section{Photoelastic bench: - (FIG 9)}

The photoelastic bench consisted of a circular polariscope with a white light source was used. Each model was placed in a polariscope and loaded at $100 \mathrm{~N}$ force at an angle of $45^{\circ}$. The value was digitally controlled by a button which increased or decreased the weight. Colourful fringes called isochromatic fringes were seen in the photoelastic material after loading. The fringes were seen in the dark field set up. A hand held digital camera was used to capture the fringe pattern onto the film. These fringes are now ready for measurement.

\section{Photoelastic interpretation: -}

The total number of isochromatic fringes observed fringes observed is directly proportional to the stress in the photoelastic resin model, and fringe crowding represents areas of high stress concentration. "Fringe order" is the numerical value assigned to an observed fringe based on its position in the colour sequence.

Sequence of coloured fringes produced in a dark field with a white light is as follows:-

\begin{tabular}{|c|c|}
\hline Colour & Fringe order \\
\hline Black & 0.00 \\
\hline Grey & 0.28 \\
\hline White & 0.45 \\
\hline yellow & 0.60 \\
\hline Orange & 0.79 \\
\hline Red & 0.90 \\
\hline Tint of passage no 1 & 1.00 \\
\hline Blue & 1.06 \\
\hline Blue-green & 1.20 \\
\hline Green-yellow & 1.38 \\
\hline Orange & 1.62 \\
\hline Red & 1.81 \\
\hline Tint of passage no 2 & 2.00 \\
\hline Green & 2.33 \\
\hline Green-yellow & 2.50 \\
\hline Pink & 2.67 \\
\hline Tint of passage no 3 & 3.00 \\
\hline Green & 3.10 \\
\hline Pink & 3.60 \\
\hline Tint of passage no 4 & 4.00 \\
\hline Green & 4.13 \\
\hline
\end{tabular}

The tint of passage is a sharp dividing zone between red and blue in the first-order fringe, red and green in the second-order fringe and pink and green in the third order fringes. The first tint of passage corresponds to fringe order one, the second tint of passage to fringe order two, and so on. Analysis with white light is limited to the first five fringes because the colours become pale and are difficult to distinguish beyond that point. In cases where reading beyond the 5th fringe order is required, a monochromatic light source is used in which isochromatics appear as black and white bands.

The colour fringe pattern and order in the alveolar bone and the tooth is noted down. 
Four Basic Technique Of Photoelasicity

\section{Discussion}

* Two dimensional photoelasticity: - A two dimensional model is studied.

* Three dimensional photoelasticity: - A three dimensional model is made and stresses are frozen in this model.

* Photoelastic coating analysis: - thin coating of double refracting plastic is cemented to the surface of the model and only surface can be obtained.

* Digital photoelasticity: - new technique wherein the fringes are calibrated in a digital polariscope to minimize human error.

\section{Commonly Used Photoelastic Material}

1. Epoxy resin

2. Araldite CT 200 (RESIN) / HT901(HARDENER)

3. Araldite CY230 (RESIN) /HY951 (HARDENER)

4. polycarbonate

5. Glass

6. Celluloid

7. Urethane rubber

8. Catalin

9. Colombia resin CR-39

10. Homalite

\section{Polariscope}

The optical instrument used in photoelastic stress analysis is called polariscope. The observations are in the form of optical fringes called Isoclinics and Isochromatics, which are formed when the photoelastic model is placed in its field and loaded.

Different types of polariscope are: -

* Transmission polariscope

* Reflection polariscope

* Scattered light polariscope

These are further seen two arrangements: -

$>$ Plane polariscope

Circular polariscope

Stresses are produced as a result of mastication forces imposed on a structure. The distribution or pattern of these stresses is a result of the angle of the load and the geometry of the object.

Photoelastic stress analysis involves applying a given stress rate to a model and utilizing the induced birefringence of the material to examine the stress distribution within the model. The magnitude and direction of stress at any point can be determined by examining the fringe pattern. In this study photoelastic analysis was carried out to obtain a more authentic result.

This article provides an easy and economical technique for the fabrication of a photoelastic model. The material used is easily available. The procedure of manipulation of the material is not very technique sensitive and the result obtained is appreciable. Hence it can be used for the study of stress distribution in the tooth and alveolar bone.

\section{Conclusion}

This article provides with a simple technique and a cost effective material to make an accurate model of the teeth. An accurate model helps provide an excellent fringe pattern to determine the stress pattern and distribution on the object of interest thus providing an optimum result.

\section{References}

[1] Rao Venkateswara P, Rao Kannaji A, Rao Kameswara T.V. Araldites for Photo-Elastic Studies and Their Transition Temperatures Jpn. J. Appl. Phys. 13 (1974) pp. 1199-1202

[2] Agarwal R.B, Teufel L.W. Epon 828 epoxy: A new photoelastic-model material Experimental Mechanics Volume 23, Number 1, 3035

[3] Badran Serene A., Orr John F., Stevenson Mike and Burden Donald J. Photo-elastic stress analysis of initial alignment arch wires. Eur J Orthod 25 (2003) 117-125

[4] Freeman Patricia W., Weins William N. Puncturing Ability of Bat Canine Teeth:The Tip. Mammalogy Papers: University of Nebraska State Museum(1997)

[5] Srinivasan Murali, Padmanabhan TV. Intrusion in implant-tooth-supported fixed prosthesis. An invitro photoelastic stress analysis. IndianJDentRes19(1)2008 
[6] F. De Candia, R. Russo, V. Vittoria. Mechanical and photoelastic properties of ethylene-propylene copolymers related to chain microstructure J. Phys. Chem., 1976, 80 (27), pp 2961-2966

[7] Eric F. Finlayson. Stress intensity factor distributions in bimaterial systems - a three-dimensional photoelastic investigation. Faculty of the Virginia Polytechnic Institute and State University. February, 1998 Blacksburg, Virginia.( dissertation).

[8] Amarante Vasconcellos Martha, Pereira Soares Venicius Marcos, Darwish Ibrahim Aref Fathi, Arnaldo Freitas Camarao. Virtual analysis of stresses in human teeth restored with esthetic posts. Materials Research 2008;11(4):459-463.

[9] Lupke. M, Gardemin. M, Kopke. S, Seifert. H, Staszyk. C.Finite element analysis of the equine periodontal ligament under masticatory loading.Wien. Tierazil. Mschr- Vet.Med. Austria 2010;97:101-106.

[10] Pierrisnard Laurent etal. Corono-radicular reconstruction of pulpless teeth: A mechanical study using finite element analysis. J Prosthet Dent 2002;88:442-8.

\section{FIGURES}

\section{FIG 1: -}

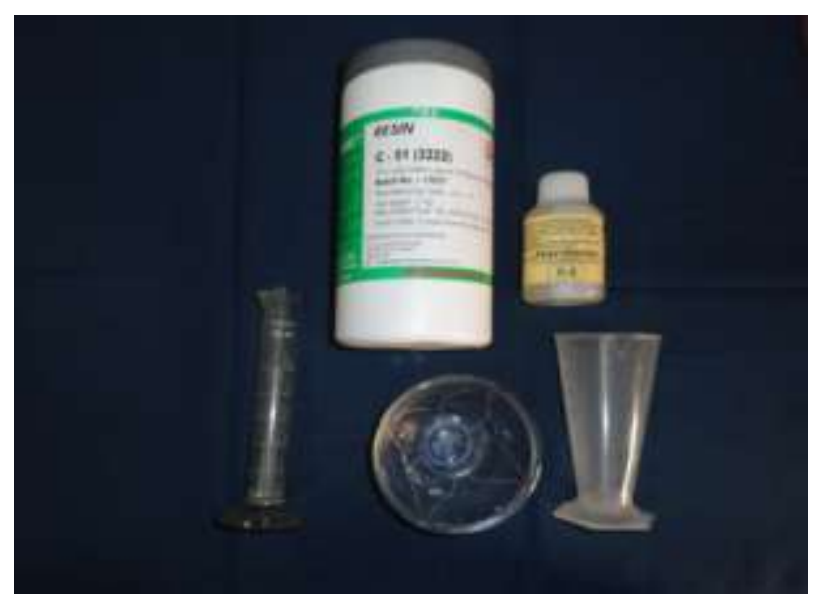

Figure 1:- Armamentarium for study

\section{FIG 2: -}

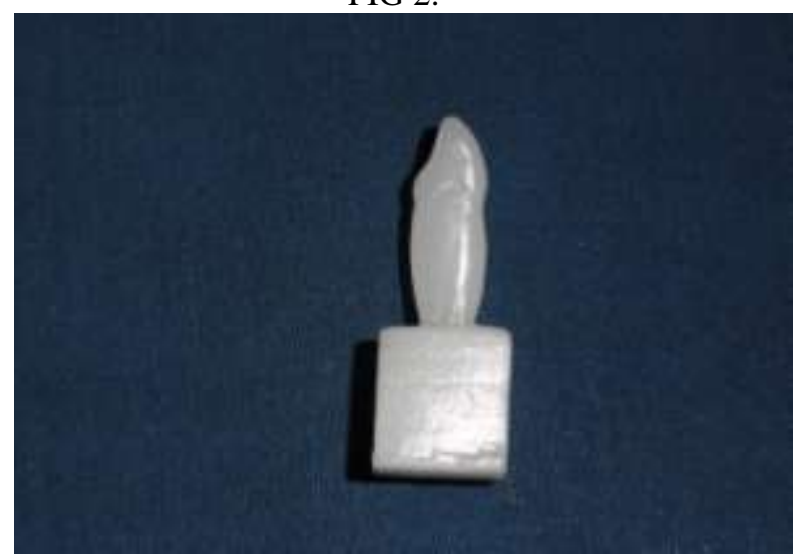

Figure 2:- Wax model of maxillary central incisor.

FIG 3:-

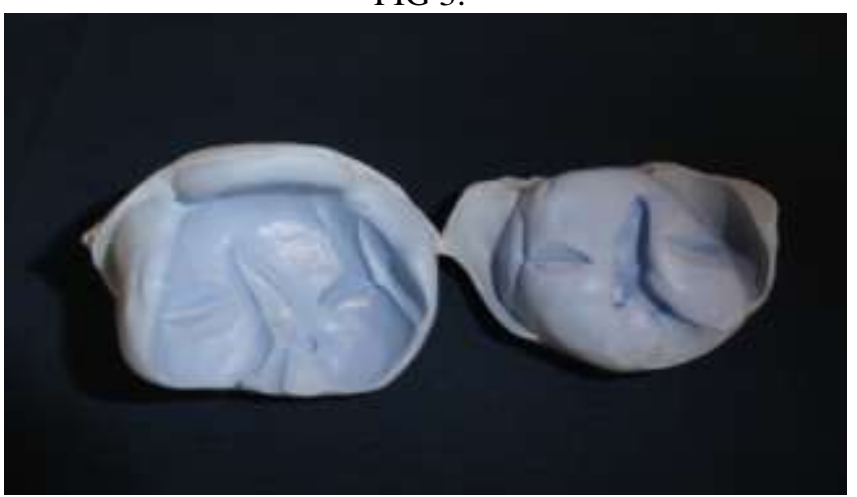

Figure 3:- putty mould of the tooth. 


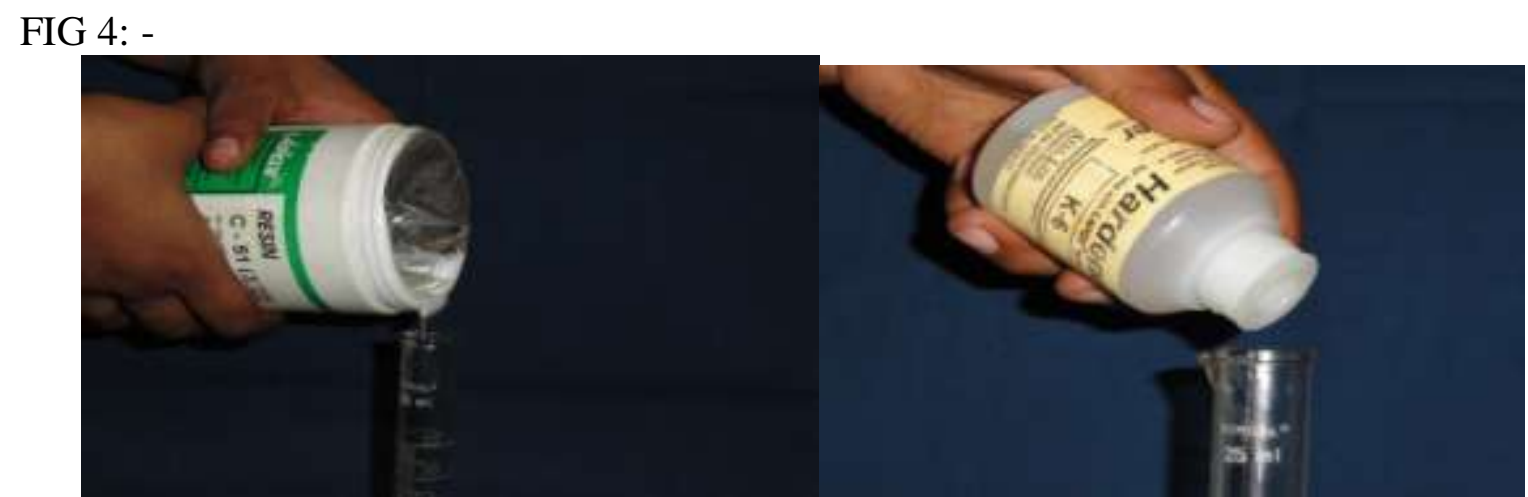

Figure 4:- Resin and hardener dispensed to the beaker.

FIG 5: -

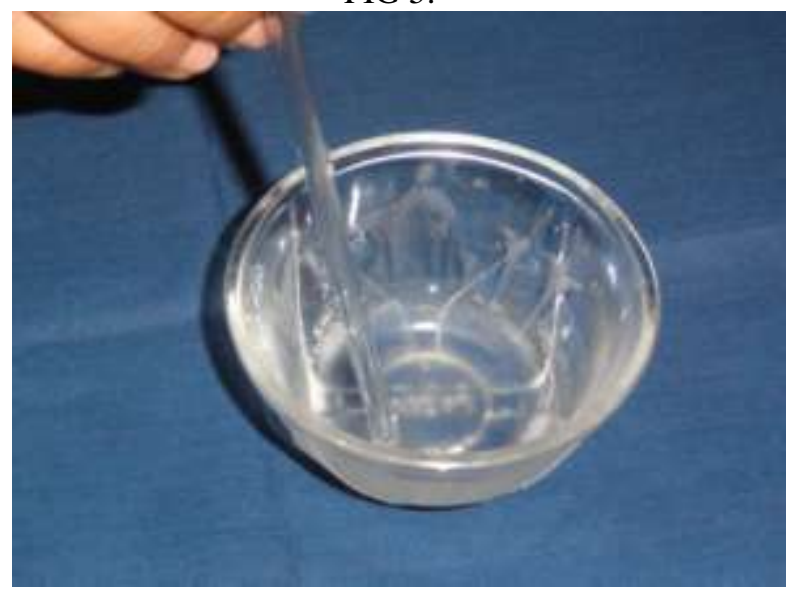

Figure 5- Mixing of epoxy resin.

FIG 6: -

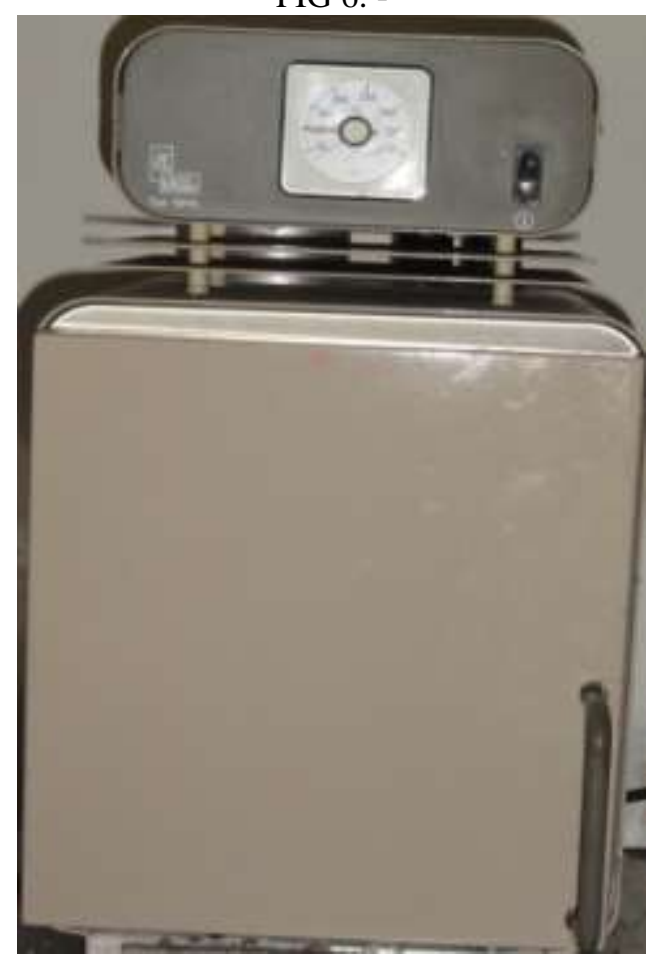

Figure 6:- Preheating unit. 
FIG 7:-

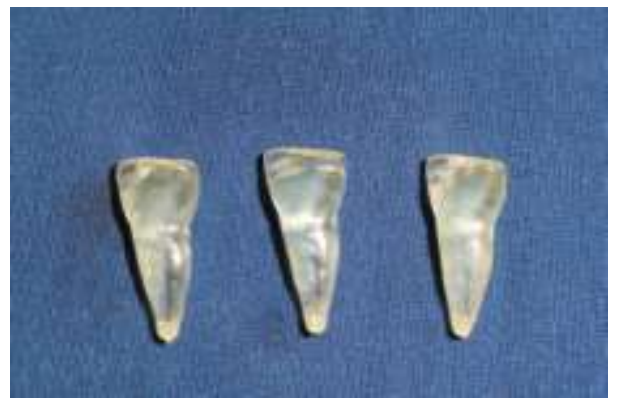

Figure 7:- Photoelastic model of tooth.

FIG 8: -

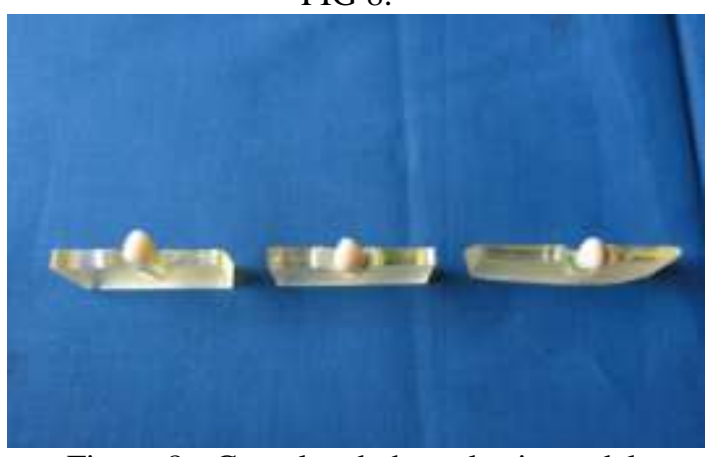

Figure 8:- Completed photoelastic model.

FIG 9: -

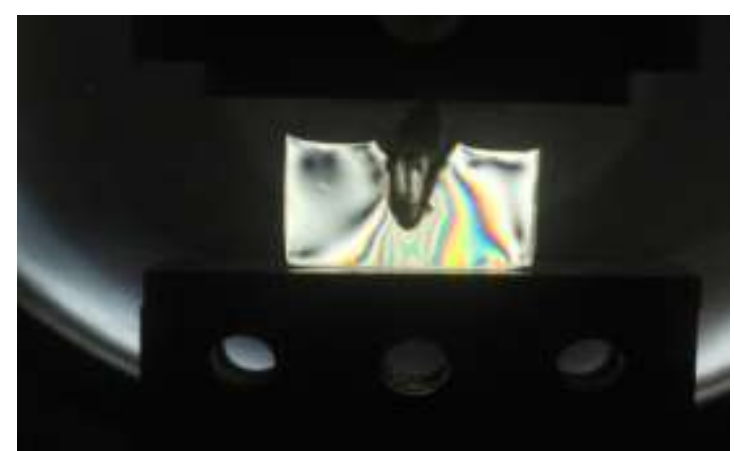

Figure 9:- fringes are appreciated in the model.

Figure 10:-

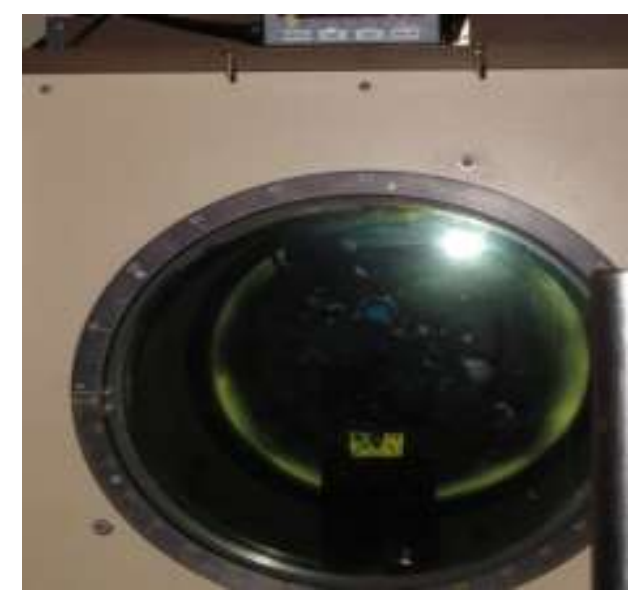

Figure 10:- photoelastic bench with the model. 